\title{
Clinical Utility of Shear Wave Elastography Patterns for Differentiating between Benign and Malignant Breast Lesions
}

\author{
Chang Shin Jung, Sang Hyup Lee, Hyun Yul Kim, Hyun Jun Paik, Kyung Jin Nam ${ }^{1}$, Hong Jae Jo², Youn Joo Jung \\ Departments of Surgery and ${ }^{1}$ Radiology, Pusan National University Yangsan Hospital, Yangsan; ${ }^{2}$ Department of Surgery, Pusan National University \\ Hospital, Busan, Korea
}

Purpose: This retrospective study evaluated the utility of shear wave elastography (SWE), Tozaki's visual pattern classification, and conventional Breast Imaging Reporting and Data System (BI-RADS) classification for differentiating between benign and malignant lesions. Methods: Between May 2015 and July 2016, 388 patients underwent SWE and B-mode ultrasonography. The BI-RADS system was used to exclude cases with category 1-2 lesions or unbiopsied category 3 lesions. A total of 100 patients with 100 solid breast masses underwent tissue sampling (ultrasonography-guided core biopsy or vacuum-assisted biopsy) or surgical excision. The quantitative elasticity was measured for each lesion, and the imaging and histological findings were compared. Results: The mean age of the patients was 51 years (range, 18-79 years). Histological examination identified 50 malignant lesions and 50 benign lesions. According to the BI-RADS classification, 20 lesions were classified as category 3, 56 as category 4, and 24 as category 5 . Based on the Tozaki classification, 39 lesions were classified as pattern 1 , seven as pattern 2, 23 as pattern 3, and 31 as pattern 4 . If patterns 1 and 2 were assumed to be benign, and patterns 3 and 4 were assumed to be malignant, the combination of BI-RADS and SWE provided a sensitivity of $100 \%$ (50/50), a specificity of $92.0 \%$ (46/50), a positive predictive value of $92.5 \%$ (50/54), and a negative predictive value of $100 \%$ (50/50). Conclusion: The combination of SWE and BIRADS was useful for evaluating breast lesions, improved the specificity of ultrasonography and may help facilitate appropriate treatment planning.

Key Words: Breast neoplasms, Elasticity imaging techniques, Ultrasonography

\section{INTRODUCTION}

Ultrasonography (US) and mammography are useful breast examination techniques, although mammography has limited sensitivity for women who are young or who have dense breasts. Thus, US remains important for detecting and classifying breast lesions for these women. Breast lesions can be categorized using the guidelines from the American College of Radiology and the Breast Imaging Reporting and Data System (BI-RADS) [1], which indicate the need for follow-up and/or treatment in each category.

Elastography has recently been used to distinguish between benign and malignant breast lesions using qualitative and quantitative mea-

Correspondence: Youn Joo Jung

Department of Surgery, Pusan National University Yangsan Hospital, 20 Geumoro, Mulgeum-eup, Yangsan 50612, Korea

Tel: +82-55-360-2124, Fax: +82-55-360-2154, E-mail: gsjyj@hanmail.net

This work was supported by a 2-year research grant from Pusan National University.

Received: Aug 31, 2017 Revised: Sep 23, 2017 Accepted: Nov 26, 2017 sures of lesion stiffness. The current techniques are strain imaging and shear wave elastography (SWE). Strain elastography uses Young's modulus to calculate the degree of tissue deformation during direct manual compression [2,3] because rigid structures (e.g., a rigid tumor) are less likely to deform. However, its reliance on manual compression is prone to inter- and intra-observer variation. Moreover, local deformation can be affected by the characteristics of the surrounding tissues.

SWE was developed to overcome these shortcomings by evaluating mechanical vibrations that are generated using an acoustic radiation force. A very fast (5,000 frames/s) US acquisition sequence is used to capture the propagation of shear waves [4,5], which can be depicted in real-time as a color-coded stiffness map. Furthermore, SWE can be used to calculate the minimum, mean, and maximum elasticities in the region of interest (ROI). As the acoustic radiation force is created by the US probe, this technique provides more consistent results compared to strain elastography.

The present study aimed to evaluate the utility of SWE in clinical 
practice, using Tozaki's visual pattern classification, and whether the combination of SWE and BI-RADS categorization can be used to characterize breast masses, which may help clinical decision-making among surgeons.

\section{METHODS}

\section{Patients and breast lesions}

Between May 2015 and July 2016, 388 patients underwent SWE and B-mode US, and the lesions were classified according to the BI-RADS system. A breast surgeon with 15 years of experience using breast US retrospectively performed the visual classification using Tozaki's patterns [6] and the SWE images. That surgeon was blinded to the pathological results. The visual classification, BI-RADS categorization, and pathological results were subsequently compared.

The present study examined category 3-5 lesions, as category 1-2 lesions are clearly benign, although we excluded category 3 lesions that were not biopsied. Thus, 100 women with a mean age of 51 years (range, 18-79 years) and 100 solid breast lesions were included in the analyses. All patients underwent tissue sampling (US-guided core biopsy or vacuum-assisted biopsy) or surgical excision after the US examination.

The sensitivity, specificity, positive predictive value (PPV), negative predictive value (NPV), and accuracy were calculated and compared. The McNemar test was used for paired comparison of proportions (sensitivity and specificity).

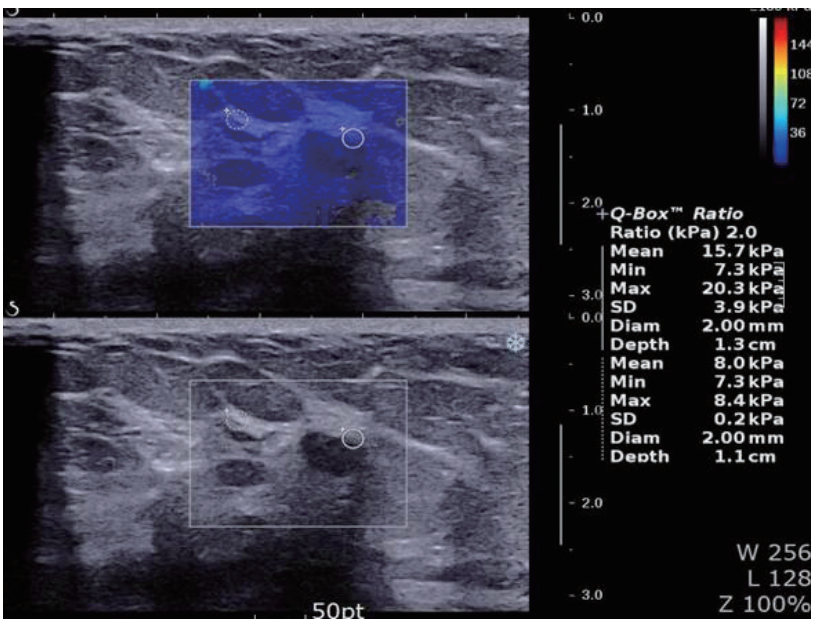

Figure 1. A 51-year-old woman with fibrocystic breast lesion. Shear wave elastography shows the lesion coded blue homogenously (pattern 1).

\section{US examinations and image evaluation}

US examinations were performed using the Aixplorer US system (SuperSonic Imagine, Aix-en-Provence, France) and linear array transducer with a bandwidth of 4 to $15 \mathrm{MHz}$. The SWE technique evaluates shear wave velocity, which is generated by a remote radiation force [7] that is created by focusing ultrasound waves using the ultrasonic probe. The radiation force displaces the tissue by a few micrometers, generating a transient shear wave in the medium. Young's modulus $(\mathrm{kPa})$ is then calculated using the shear wave velocity, and the ROI can be color-coded (red, hard areas; blue, soft areas). For the present study, the ROI was defined as the lesion and the surrounding normal tissue, and the probe was oriented perpendicular to the skin, lesion, and thoracic wall.

Tozaki's visual classification defines pattern 1 (no findings) as no difference between the margin and the interior of the lesion (homogeneously blue) (Figure 1). Pattern 2 (negative findings/artifacts) is defined as color variation at the margin or the interior of the lesion that extends beyond the lesion and continues vertically in cords along the cutaneous or thoracic wall sides (Figure 2). Pattern 3 (positive findings) is defined as a local change in color at the margin of the lesion, even if the cord-like artifacts are present (Figure 3). Pattern 4 (positive findings) is defined as heterogeneous coloring at the interior of the lesion (Figure 4)

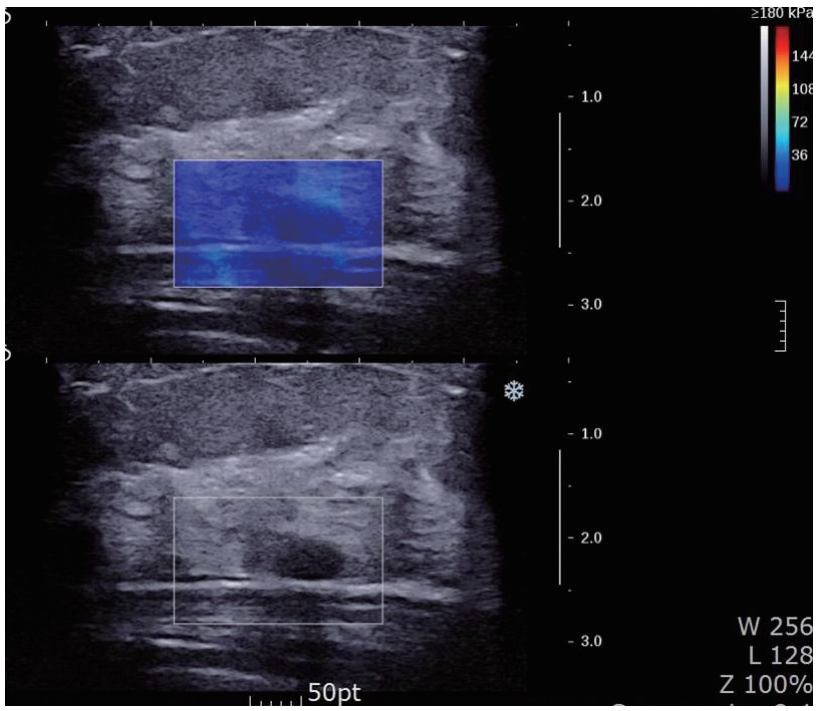

Figure 2. A 45-year-old woman with fibroadenomatous nodule. Shear wave elastography shows light green vertically striped pattern (pattern 2). 


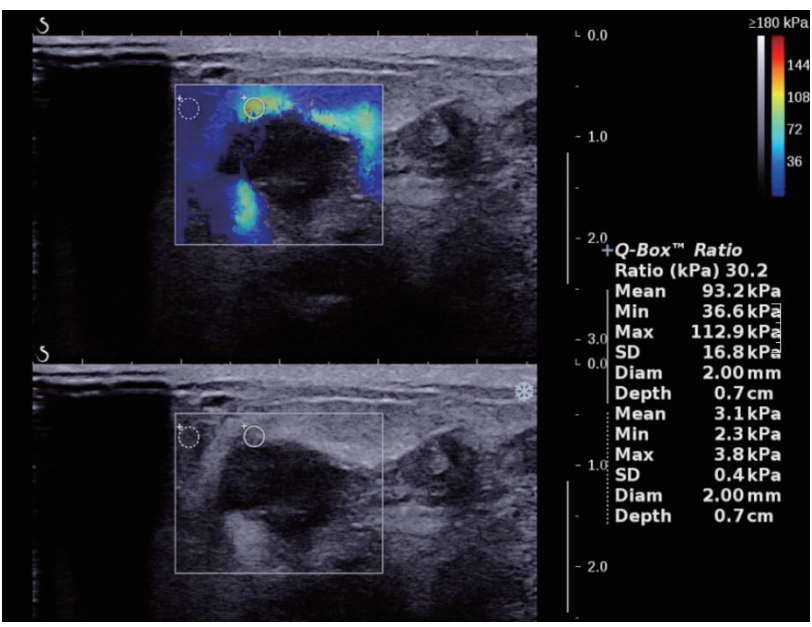

Figure 3. A 51-year-old woman with ductal carcinoma in situ. Shear wave elastography shows localized colored area at the margin of the lesion (pattern 3).

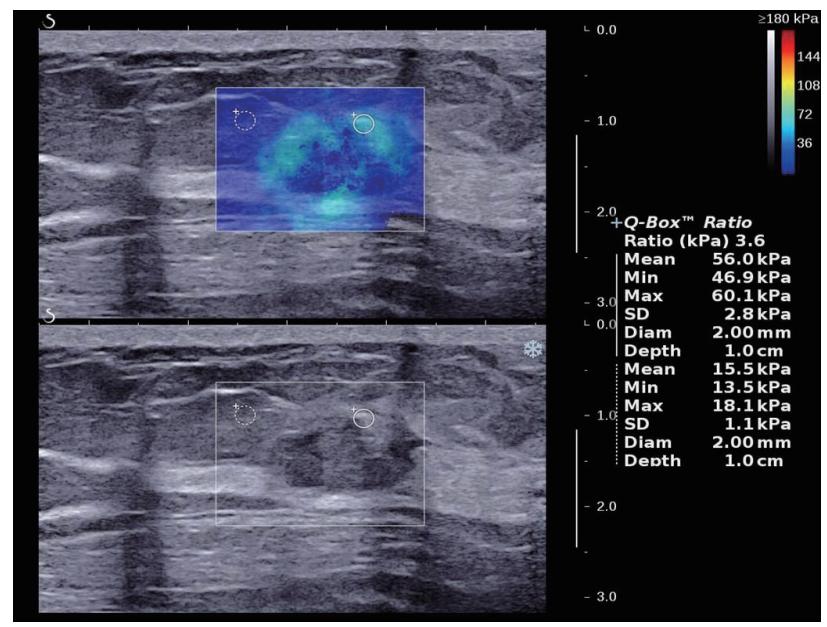

Figure 4. A 63-year-old woman with invasive ductal carcinoma. With shear wave elastography, the lesion shows heterogeneously colored areas in the interior of the lesion (pattern 4).

Table 1. BI-RADS-US category and pattern classification of SWE image

\begin{tabular}{|c|c|c|c|c|c|c|c|c|c|c|}
\hline \multirow{3}{*}{$\begin{array}{c}\text { BI-RADS-US } \\
\text { category }\end{array}$} & \multicolumn{8}{|c|}{ Pattern of SWE images } & \multirow{2}{*}{\multicolumn{2}{|c|}{ Total }} \\
\hline & \multicolumn{2}{|c|}{1} & \multicolumn{2}{|c|}{2} & \multicolumn{2}{|c|}{3} & \multicolumn{2}{|c|}{4} & & \\
\hline & Benign & Malignant & Benign & Malignant & Benign & Malignant & Benign & Malignant & Benign & Malignant \\
\hline 3 & 16 & 0 & 4 & 0 & 0 & 0 & 0 & 0 & 20 & 0 \\
\hline 4 & 23 & 0 & 3 & 0 & 3 & 8 & 1 & 18 & 30 & 26 \\
\hline 5 & 0 & 0 & 0 & 0 & 0 & 12 & 0 & 12 & 0 & 24 \\
\hline Total & 39 & 0 & 7 & 0 & 3 & 20 & 1 & 30 & 50 & 50 \\
\hline
\end{tabular}

BI-RADS-US = Breast Imaging Reporting and Data System-ultrasonography; SWE = shear wave elastography.

\section{RESULTS}

The histopathological results indicated that 50 lesions were malignant and 50 lesions were benign. The malignancies included ductal carcinoma in situ $(\mathrm{n}=1)$, invasive ductal carcinoma not otherwise specified ( $\mathrm{n}=46)$, mucinous carcinoma $(\mathrm{n}=1)$, and invasive lobular carcinoma $(\mathrm{n}=2)$. The 50 benign lesions included fibroadenoma $(n=14)$, benign proliferative disease $(n=33)$, benign phyllodes tumor $(\mathrm{n}=2)$, and atypical ductal hyperplasia $(\mathrm{n}=1)$. Table 1 shows the results from the visual classification, which included pattern 1 for 39 lesions, pattern 2 for seven lesions, pattern 3 for 23 lesions, and pattern 4 for 31 lesions. Based on the assumption that patterns 1 and 2 were benign and patterns 3 and 4 were malignant, the SWE analysis provided a sensitivity of $100 \%$ (50/50), a specificity of $92.0 \%$ (46/50), a PPV of 92.5\% (50/54), a NPV of $100 \%$ (46/46), and an accuracy of $96.0 \%$ $(96 / 100)$. No false-negative malignant lesions were found, and the histological types of the four false-positive benign lesions were fibroade-
Table 2. Diagnostic performance characteristics: comparison BI-RADS-US category 4 lesions and pattern classification of SWE image

\begin{tabular}{lccc}
\hline Variable & $\begin{array}{c}\text { BI-RADS-US } \\
(\%)\end{array}$ & $\begin{array}{c}\text { SWE } \\
(\%)\end{array}$ & $\begin{array}{c}\text { BI-RADS-US } \\
\text { and SWE (\%) }\end{array}$ \\
\hline Sensitivity & 100 & 100 & 100 \\
Specificity & 40.0 & 92.0 & 92.0 \\
Positive predictive value & 62.5 & 92.5 & 92.5 \\
Negative predictive value & 100 & 100 & 100 \\
Accuracy & 70.0 & 96.0 & 96.0 \\
\hline
\end{tabular}

BI-RADS-US = Breast Imaging Reporting and Data System-ultrasonography; $\mathrm{SWE}=$ shear wave elastography.

noma $(\mathrm{n}=3)$ and phyllodes tumor $(\mathrm{n}=1)$ (Table 2$)$.

Based on the BI-RADS-US categories, the rates of breast cancer were $0 \%$ for category $3(0 / 20), 46 \%$ for category $4(26 / 56)$, and $100 \%$ for category 5 (24/24) (Table 1). A cutoff point between BI-RADS-US categories 3 and 4 provided a sensitivity of 100\% (50/50), a specificity of $40.0 \%$ (20/50), a PPV of $62.5 \%$ (50/80), an NPV of $100 \%$ (20/20), and an accuracy of 70.0\% (70/100) (Table 2). Among the category 4 lesions, 26 benign lesions and no malignant lesions were assigned to pattern 1 or 
2. The combination of SWE plus BI-RADS-US category 4 provided a sensitivity of 100\% (50/50), a specificity of 92.0\% (46/50), a PPV of $92.5 \%$ (50/54), an NPV of 100\% (46/46), and an accuracy of $96 \%$ $(96 / 100)$.

\section{DISCUSSION}

Screening or examination of symptomatic breast lesions (e.g., a palpable mass) is generally performed using mammography and US. The results can be classified according to the BI-RADS system, which indicates the need for follow-up or tissue sampling. Most category 3 lesions are benign and suitable for follow-up, while category 4 lesions are strongly suspected for malignancy and are further subdivided into $4 \mathrm{a}, 4 \mathrm{~b}$, and $4 \mathrm{c}$ according to the lesion's specific status. Category 4 lesions are typically evaluated using biopsy and histological testing if possible, while category 3 lesions are not frequently evaluated using biopsy, given the low probability of malignancy. In the screening setting, confirming a suspicion of cancer is important, while suspected benign lesions can be followed in a less invasive manner (e.g., using SWE). In the present study, the BI-RADS system alone had a sensitivity of $100 \%$, but only had a specificity of $40 \%$. However, the combination of BI-RADS and SWE improved the specificity, PPV, and accuracy from $40.0 \%$ to $92.0 \%$, from $62.5 \%$ to $92.5 \%$, and from $70.0 \%$ to $96.0 \%$, respectively, $(p<0.005)$. The improved specificity indicates that some lesions (e.g., category 3 lesions, which have a malignancy rate of $<2 \%$ ) can be safely followed up without invasive examinations, such as core needle biopsy. Furthermore, Athanasiou et al. [2] have reported that B-mode US had a specificity of only $63 \%$ for solid breast lesions, although the specificity increased to 96\% when SWE was applied. Thus, BI-RADS category 4 lesions can be down-staged to category 3 , or category 3 lesions can be down-staged to category 2 .

In addition to improving the accuracy of US, SWE is easy to perform because it uses the same ultrasonic probe. Furthermore, the results can be color-coded, similar to color Doppler imaging. Thus, aside from the quantitative data from Young's modulus, performing a rapid qualitative evaluation based on the lesion's color pattern is also possible. Moreover, SWE is more reproducible than strain elastography, and Cosgrove et al. [8] have reported that SWE provides consistent image generation and interpretation, which are reflected in its high values for intra- and inter-observer reproducibility.
During SWE, pattern 1 is easy to identify if appropriate pressure is applied to the skin and would be a good starting point for SWE training. However, the vertical cords in pattern 2 are considered an artifact, which may be the result of a deep signal extending from the chest wall to the skin, and may make it difficult to focus on a superficial ROI. Moreover, poorly controlled pressure can generate a strong signal for benign lesions. Thus, pattern 2 can be difficult to distinguish from pattern 3, and combining SWE and BI-RADS may be useful for this differentiation. Zhou et al. [9] have described pattern 3 as the "stiff rim sign" because a strong signal is observed in the mass, which supports a suspicion of malignancy. Zhou et al. [9] attributed this signal to stiffness in the surrounding tissue that is caused by cancer cells infiltrating the epithelial tissue, a low shear wave amplitude, or noise from inside the malignant lesion. Berg et al. [10] have recommend that SWE findings should not alter the treatment plan for lesions in categories 2, $4 c$, or 5 because these lesions can be improperly upstaged (category 2) or not examined using biopsy (categories $4 \mathrm{c}$ and 5), although those findings may be useful for characterizing the mass. Lee et al. [11] have classified the SWE patterns using clinicopathologic factors, lymph node involvement, grade, and vascular invasion, which helped predict the distribution of ductal carcinoma in situ. However, further research is needed to examine the clinical utility of SWE.

During the original examinations, SWE had supposedly been performed randomly, although surgeons may elect to perform SWE more frequently for cases with a high BI-RADS categorization. Thus, the present cases may not have been selected completely randomly, which is a potential limitation of this study. Visual pattern classification itself is influenced by subjective judgment. Nevertheless, we conclude that SWE is useful for breast examinations and screening. Moreover, its combination with the BI-RADS system can increase the diagnostic specificity and accuracy, which can enhance the patient's care plan.

\section{CONFLICT OF INTEREST}

The authors declare that they have no competing interests.

\section{REFERENCES}

1. Sickles E, D'Orsi C, Bassett L, Appleton C, Berg W, Burnside E, et al. 
ACR BI-RADS ${ }^{\circledR}$ Atlas: Breast Imaging Reporting and Data System. Reston: American College of Radiology; 2013. p.39-48.

2. Athanasiou A, Tardivon A, Tanter M, Sigal-Zafrani B, Bercoff J, Deffieux T, et al. Breast lesions: quantitative elastography with supersonic shear imaging: preliminary results. Radiology 2010;256: 297-303.

3. Alhabshi SM, Rahmat K, Abdul Halim N, Aziz S, Radhika S, Gan GC, et al. Semi-quantitative and qualitative assessment of breast ultrasound elastography in differentiating between malignant and benign lesions. Ultrasound Med Biol 2013;39:568-78.

4. Bercoff J, Tanter M, Muller M, Fink M. The role of viscosity in the impulse diffraction field of elastic waves induced by the acoustic radiation force. IEEE Trans Ultrason Ferroelectr Freq Control 2004; 51:1523-36.

5. Bercoff J, Tanter M, Fink M. Supersonic shear imaging: a new technique for soft tissue elasticity mapping. IEEE Trans Ultrason Ferroelectr Freq Control 2004;51:396-409.

6. Tozaki M, Fukuma E. Pattern classification of ShearWave ${ }^{\mathrm{TM}}$ Elastography images for differential diagnosis between benign and ma- lignant solid breast masses. Acta Radiol 2011;52:1069-75.

7. Tanter M, BercoffJ, Athanasiou A, Deffieux T, Gennisson JL, Montaldo G, et al. Quantitative assessment of breast lesion viscoelasticity: initial clinical results using supersonic shear imaging. Ultrasound Med Biol 2008;34:1373-86.

8. Cosgrove DO, Berg WA, Doré CJ, Skyba DM, Henry JP, Gay J, et al. Shear wave elastography for breast masses is highly reproducible. Eur Radiol 2012;22:1023-32.

9. Zhou J, Zhan W, Chang C, Zhang X, Jia Y, Dong Y, et al. Breast lesions: evaluation with shear wave elastography, with special emphasis on the "stiff rim" sign. Radiology 2014;272:63-72.

10. Berg WA, Cosgrove DO, Doré CJ, Schäfer FK, Svensson WE, Hooley RJ, et al. Shear-wave elastography improves the specificity of breast US: the BE1 multinational study of 939 masses. Radiology 2012;262:435-49.

11. Lee S, Jung Y, Bae Y. Clinical application of a color map pattern on shear-wave elastography for invasive breast cancer. Surg Oncol 2016;25:44-8. 